\title{
Education, the science of learning, and the COVID-19 crisis
}

\author{
Michael S. C. Thomas ${ }^{1}$ (D) Cathy Rogers $^{1}$
}

Published online: 25 May 2020

(C) UNESCO IBE 2020

\begin{abstract}
In the COVID-19 crisis, the science of learning has two different responsibilities: first, to offer guidance about how best to deal with the impact of the current situation, including lockdown and home-schooling; and second, to consider bigger questions about what this large-scale educational experiment might mean for the future. The first part of this Viewpoint summarises advice for parents on mental health, and on becoming stand-inteachers. The second part, taking the longer view, considers the potential negative impact of the COVID-19 crisis in increasing inequality in education; but also the potential positive impact of driving innovations in technology use for educating children.
\end{abstract}

Keywords COVID-19 $\cdot$ Learning $\cdot$ Science of learning $\cdot$ Crisis

Alongside the many new challenges posed by the COVID-19 crisis is a somewhat familiar one: how to translate scientific evidence into public policy. Politicians (in the UK at least) recite the mantra "we are led by the science", but in reality politicians, not scientists, lead. Science focuses on mechanisms, the how and the why, while politicians have the daunting job of turning that into the "so what do we do?".

On a smaller scale, the science of learning faces the same challenge. How can a scientific understanding of teaching and learning inform the much broader canvas of education policy and educational practices? More specifically, how can the slow, cumulative knowledge built up through research translate to meet classroom teachers' immediate needs?

Michael S. C. Thomas

m.thomas@bbk.ac.uk

Cathy Rogers

cathyjanerogers01@gmail.com

1 Centre for Educational Neuroscience, Birkbeck, University of London, Malet Street,

London WC1E 7HX, UK 


\section{The current crisis}

In the current crisis, with schools closed, children at home, and parents expected to take on teaching duties, psychology and the science of learning have two different responsibilities. The first is to offer guidance about how best to deal with the current situation. The second is to consider bigger questions about what this large-scale educational experiment might mean for the future.

\section{Dealing with the current situation}

The Association for Psychological Sciences (APA 2020) has summarised advice on the social impact of lockdown for both children and adults, as well as considering the implications of the increased anxiety produced by the pandemic.

Some of the main points concerning mental health include:

- Loneliness can make you ill by increasing stress, which can severely impact physical and mental health. While epidemiological models are good at measuring tangibles such as infection and mortality rates, they are less able to measure social costs such as loneliness. The consequences are no less real for those suffering.

- Parents' own mental health is important for children's development since caregivers are children's primary emotion regulators in times of stress. Parents need to look after their own mental health, for the sake of their children as well as themselves.

- Symptoms of anxiety and depression correlate with level of media exposure. Since this pandemic saturates us with information 24 hours a day, it is recommended that adults choose only one or two times a day to watch or read news updates. Parents also need to be aware of the information passed on to children, both factual and emotional, because children will learn from whatever information is around them.

- Changing behaviour is hard, particularly when the consequences seem quite abstract (e.g., don't scratch your nose because that'll protect your grandpa). Gaining knowledge about how things work can help. For younger children, rule-based information such as "wash your hands" is much less effective without explaining why and how it works (reducing illness transmission, staying healthy).

Similarly, for those who have become stand-in-teachers overnight, the science of learning can provide some general pointers:

- Remember that learning can be slow and difficult; maintaining motivation and minimising frustration is key.

- Make learning meaningful to the child: what do they want to learn about? For younger children, follow their lead. For older children, who are following stricter learning roadmaps, try to relate topics in subjects they don't like to topics in subjects they do, e.g., relate testing for chlorine gas in Chemistry to a History lesson on the use of chlorine as a weapon in the First World War.

- Promote autonomy by giving children choice and flexibility. Try encouraging them to create their own timetables, including breaks and snack times. 
- Give frequent, positive verbal feedback; it acts as a powerful reward which can promote better learning.

- Most important of all, physical exercise, a good diet, and sufficient sleep are the cornerstones of any effective learning.

\section{Implications for the future of education}

We all hope that COVID-19's negative impact on education will be short-lived. But the reality is that many countries in the world are now participants in the biggest unplanned experiment that education has ever seen. What are some of the possible outcomes?

On the downside, the requirement for children to continue their studies at home may exacerbate differences in educational progress caused by inequalities. Children from homes with fewer resources and less opportunity for parental support risk falling behind, widening pre-existing gaps. Worse still, some children may feel unsafe in their homes. Children of well-off parents often live in bigger houses, have their own bedrooms, support from two parents rather than one, and better access to technology, books, and other learning resources, not to mention basics such as food. At least in school, pupils share the same spaces, lessons, teachers, and resources, which levels opportunities to some extent. Moreover, studying at home will affect some age groups more than others. Teenagers, whose emotional development propels them to move away from parents and carers and towards friends and peers, are likely to be more adversely affected.

On the potential upside, the new forced reliance on technology in education may accelerate some changes that had already begun. Might the pandemic cause educators to think harder about the current models of educational provision, learning faster about which uses of technology are successful and which are not? Granted, the initial versions of education at home often look quite like the traditional teacher-led classroom, with teachers setting work to be completed at home, supplemented by some websites or online tutoring for narrow skills such as spelling or arithmetic. But perhaps there are also initial glimpses of what a technology-led revolution in education might look like: teachers serving as curators for virtual learning environments, which children can explore together with classmates to solve problems. In these environments, children can self-regulate their learning, switching to video tutorials or guidance from teachers when they need to learn new skills to proceed. Think Minecraft, Roblox, and YouTube.

Of course, this kind of revolution is some way off. Perhaps more importantly, returning to our opening theme of the role of policymakers, policy is not the only driver of these innovations. Instead, progress is driven by the cultural and commercial technology ecosystem. This crisis has made it apparent that school-provided IT systems for computerbased learning are becoming redundant. They are too expensive, too cumbersome, and too quickly out of date. Perhaps we need instead to move to personal devices integrated into schools. Just as we turn on Airplane Mode when we get on a plane, perhaps we will turn on Education Mode when we enter school.

This suggests the policy solutions needed to reshape education through technology are not prescriptive, but regulatory. Policy needs to incentivise commercial developers to move into education. The role of policymakers, by contrast, should be to understand and mitigate the possible risks of technology use in children, as documented by research. As a starting point, these risks include screen time addiction and intolerance of boredom, displacement 
of other activities (e.g., homework, play), social media bullying, unreliable information, sleep disruption, violent or otherwise age-inappropriate content, and data protection issues.

The wholehearted engagement of teenagers with computer games demonstrates that such games can, in principle, be powerful tools for affecting behaviour. Deliberate attempts to create games that deliver learning - to gamify education - have so far been relatively poor because their focus has been on content and not game mechanics. Such mechanics, triggering engagement and curiosity in children, are understood much better by wellresourced commercial game developers. This is where government incentivisation may be effective, to encourage commercial developers to produce engaging and powerful educational games and learning environments.

One can hope that, despite the many dark sides of the current pandemic, there might also be a sliver of silver lining for education.

\section{References}

APA [American Psychological Association] (2020). Human behavior in the time of COVID-19: Learning from psychological science. https://www.psychologicalscience.org/observer/human-behavior-in-thetime-of-covid-19.

Thomas, M. S. C., Mareschal, D., \& Dumontheil, I. (2020). Educational neuroscience: Development across the lifespan. London: Routledge.

Publisher's Note Springer Nature remains neutral with regard to jurisdictional claims in published maps and institutional affiliations.

Michael S. C. Thomas (United Kingdom) is a professor of cognitive neuroscience at Birkbeck, University of London, and Director of the University of London Centre for Educational Neuroscience (http://www. educationalneuroscience.org.uk/). His work focuses on translational research and the dialogue between educators, psychologists, and neuroscientists. He has just edited a new book on this topic, Educational Neuroscience: Development Across the Life Span (Routledge, 2020).

Cathy Rogers (United Kingdom) is completing her doctoral studies in educational neuroscience, investigating the relationship between the development of cognitive control skills and creativity in children. Previously, she spent twenty years working as a producer, presenter, and creative director in the TV industry. 